\title{
Radioactivité naturelle des aliments ${ }^{\star}$
}

\author{
L. JEANMAIRE ${ }^{\star \star}$ \\ (Manuscrit reçu le 21 décembre 1984)
}

RESUME

\begin{abstract}
Les radionucléides dits " naturels" sont présents dans l'alimentation en proportions variables selon la nature et l'origine des aliments. Après un rappel des principes permettant de comprendre les transferts dans le milieu, les radionucléides présents dans l'alimentation sont examinés individuellement.
\end{abstract}

\section{ABSTRACT}

The so-called " natural" radionuclides are present in food in different proportions according to the nature and origin of the food products. After a summary of the principles allowing to understand environmental transfers, a study is made of the respective radionuclides to be found in food.

L'alimentation provient de l'environnement qui contient les radionucléides dits naturels. De ce fait, les radionucléides sont présents en proportions variables selon la nature et l'origine des aliments. Les pratiques culinaires peuvent entraîner des modifications. Après avoir rappelé quelques principes permettant de comprendre les transferts dans le milieu, les radionucléides présents dans l'alimentation seront examinés individuellement.

1. Les aliments sont constitués de matière organique d'origine végétale ou animale, d'eau, de substances minérales.

1.1. Les végétaux puisent une partie de l'eau et les substances minérales directement dans le sol ; la cellule végétale choisit, dans le milieu, les éléments utiles à son métabolisme ; ce sont en majorité des éléments tels que les alcalins, les alcalino-terreux, le soufre, le phosphore... La composition minérale de la cellule végétale diffère donc de celle du milieu. L'atmosphère est également une source unique ou partielle d'éléments constitutifs du tissu végétal ; c'est le cas du carbone et de l'eau.

Cette composition interne d'origine métabolique est modifiable sous des influences extérieures. Les végétaux à grande surface foliaire reçoi-

* Communication présentée lors des Journées sur "les données actuelles sur la radioactivité naturelle" organisées par la Société française de Radioprotection à Monte-Carlo, du 5 au 7 novembre 1984.

** Commissariat à l'énergie atomique, Institut de protection et de sûreté nucléaire, Département de protection sanitaire, Service d'études appliquées de protection sanitaire, BP 6, 92260 Fontenay-aux-Roses (France). 
vent des poussières provenant de la mise en suspension de fines particules terrestres par le vent. En outre, d'autres éléments d'origine terrestre (comme les descendants du gaz radon) ou d'origine cosmique existent normalement dans l'atmosphère et retombent au sol où ils peuvent se déposer également sur les végétaux. La pluie peut projeter de la terre sur les végétaux tels que salades et fraises. Enfin, certains végétaux sont récoltés dans le sol. Toutes ces causes vont apparemment modifier la composition interne d'un végétal. Selon le soin qui préside à la culture et à la transformation de ces végétaux en aliment, la part de la contamination externe sera plus ou moins grande.

1.2. La cellule animale, comme la cellule végétale, choisit les éléments dont elle a besoin avec des critères modulés selon le type de tissu auquel elle appartient. Par exemple, il y a concentration du potassium intracellulairement, du sodium dans les liquides extracellulaires, du calcium dans le tissu osseux, etc. En considérant l'animal dans sa totalité, le même phénomène de contamination extérieure peut modifier la composition naturelle réglée par le métabolisme des différents tissus. C'est ainsi que le contenu digestif ou les particules retenues dans d'autres organes filtrants tels que poumon, branchies, n'ont probablement pas la composition chimique moyenne de l'animal. La composition de l'aliment d'origine animale sera soumise à plusieurs influences: position occupée par l'espèce dans la chaîne trophique, partie comestible, pratiques culinaires, etc.

2. Pour l'homme, l'irradiation d'origine alimentaire représente environ $15 \%$ de l'irradiation naturelle totale et, dans les conditions normales, plus de $80 \%$ proviennent de deux radionucléides - le potassium 40 et le polonium 210 - alors que de nombreux éléments sont présents dans les aliments. Le Comité scientifique des Nations-Unies pour l'étude des effets des rayonnements ionisants (UNSCEAR) [1] les répartit en deux groupes de radionucléides : celui des radionucléides cosmogéniques produits par les rayons cosmiques et celui des radionucléides prépondérants présents dans l'écorce terrestre (tableau I). Ce classement sera utilisé pour les besoins de l'exposé. La quantité ingérée annuellement donnée pour chaque radionucléide a été calculée pour un régime type. Elle représente une estimation assez grossière plutôt qu'une valeur exacte.

\subsection{Radionucléides cosmogéniques}

Quatre radionucléides produits par le rayonnement cosmique contribuent de façon appréciable à l'irradiation interne : le carbone 14 , le tritium, le béryllium 7 et le sodium 22 .

2.1.1. Le carbone 14 est le plus important de ces radionucléides car il marque tout le carbone biologique. On admet que l'activité naturelle du carbone est de 227 Bq. $\mathrm{gg}^{-1}$ de carbone stable. Elle a diminué au cours de ce siècle par dilution avec le gaz carbonique des combustibles fossiles utilisés en grande quantité (effet Suess). Le ${ }^{14} \mathrm{C}$ est également produit lors des explosions nucléaires et, en 1965, les mesures ont montré que l'excédent de ${ }^{14} \mathrm{C}$ dans la troposphère était de $70 \%$. II est présent dans tous les aliments à la même activité spécifique que celle du milieu sous forme de matière organique. La quantité annuelle ingérée est de $20 \mathrm{kBq}$.

2.1.2. Le tritium naturel est présent de façon universelle sous forme d'eau tritiée à une concentration estimée à $400 \mathrm{~Bq} \cdot \mathrm{m}^{-3}$ pour les eaux de 
surface continentale. Le tritium est également produit dans les explosions nucléaires et dans l'industrie, à un niveau tel que ce tritium artificiel est supérieur de plusieurs ordres de grandeur au niveau naturel. Le tritium est présent dans tous les aliments sous forme d'eau et de matière organique tritiées. L'ingestion annuelle de tritium naturel est de $360 \mathrm{~Bq}$.

TABLEAU I

Activité comparée de radionucléides dans le sol, l'alimentation et les tissus humains

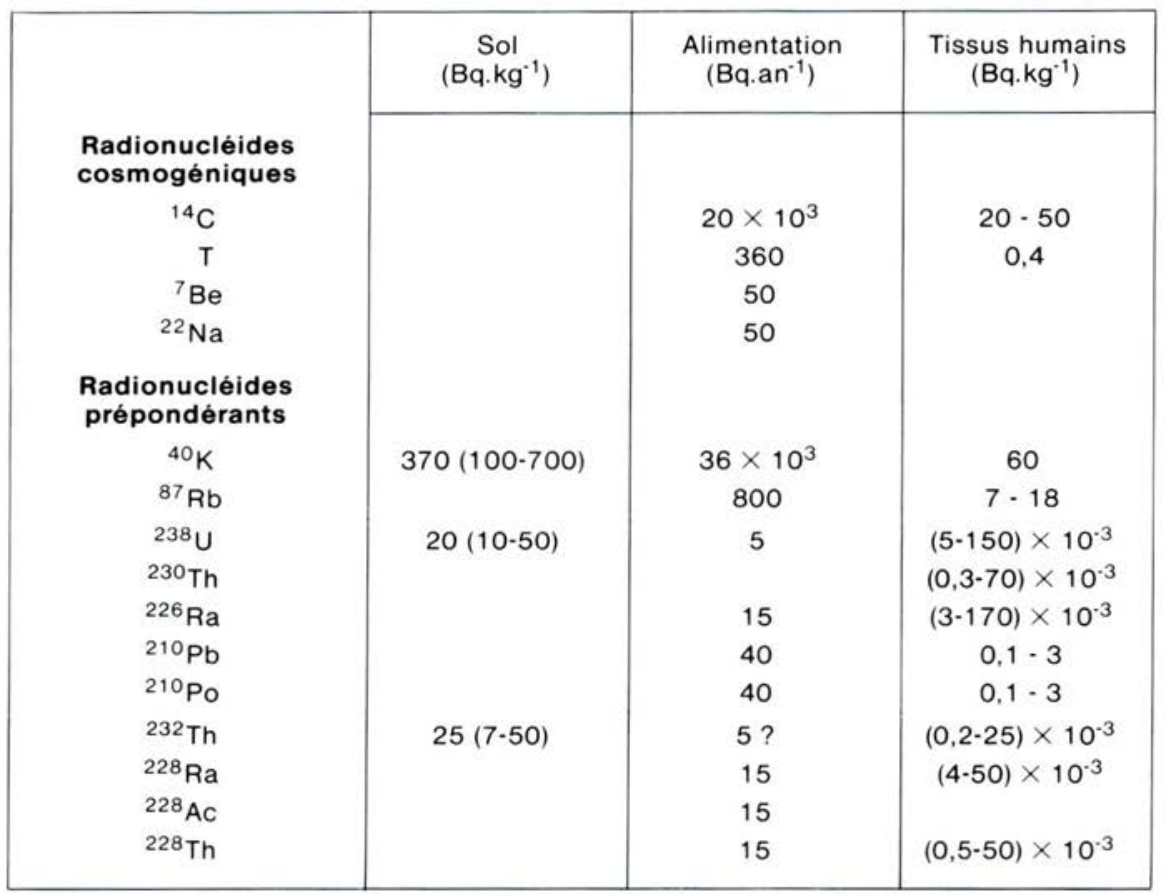

2.1.3. Le béryllium 7 provient surtout des végétaux à grande surface foliaire sur lesquels il se dépose. II existe également dans les moules, sans doute retenu par les organes de filtration de ce mollusque. L'ingestion annuelle est de $50 \mathrm{~Bq}$.

2.1.4. La quantité ingérée annuellement de sodium 22 serait également de $50 \mathrm{~Bq}$.

\subsection{Radionucléides prépondérants}

Ils comprennent des éléments isolés comme le potassium 40 et le rubidium 87 , ainsi que les éléments des familles de l'uranium et du thorium naturels.

2.2.1. Le potassium 40 est l'élément qui habituellement provoque la plus forte irradiation interne d'origine naturelle. Le potassium est un 
constituant normal et indispensable de la matière vivante. II est présent dans les aliments, quelle que soit leur origine. L'ingestion annuelle est de $36 \mathrm{kBq}$.

2.2.2. La littérature indique les valeurs du rubidium 87 dans les tissus humains, mais il y a peu d'information sur le rubidium dans l'alimentation. Cependant, quelques mesures effectuées en France sur les rations alimentaires permettent d'estimer l'ingestion annuelle à $800 \mathrm{~Bq}$.

\subsubsection{Série de l'uranium ${ }^{238} \mathrm{U}$}

Cette série, comprenant quinze radionucléides principaux, a été divisée par I'UNSCEAR en cinq sous-familles, dans lesquelles l'activité du précurseur contrôle en grande partie celle des descendants :

1) ${ }^{238} \mathrm{U}$ à ${ }^{234} \mathrm{U}$; 2) ${ }^{230} \mathrm{Th}$; 3) ${ }^{226} \mathrm{Ra}$; 4) ${ }^{222} \mathrm{Rn}$ à ${ }^{224} \mathrm{Po}$; 5) ${ }^{210} \mathrm{~Pb}$ à ${ }^{210} \mathrm{Po}$.

2.2.3.1. Sous-famille ${ }^{238} \mathrm{U}:{ }^{238} \mathrm{U},{ }^{234} \mathrm{Th},{ }^{234} \mathrm{~m} \mathrm{~Pa},{ }^{234} \mathrm{U}$. La présence de ces éléments dans l'alimentation est, sans doute, d'origine externe, par dépôt foliaire ou un autre mécanisme. Dans l'eau, la teneur en uranium est généralement faible, mais certaines eaux françaises ont des concentrations en ${ }^{238} \mathrm{U}$ de l'ordre de $2 \mathrm{~Bq} \cdot \mathrm{I}^{-1}$, avec de surcroît un rapport ${ }^{234} \mathrm{U} /{ }^{238} \mathrm{U}$ bien supérieur à 1 . L'ingestion annuelle dans une région à radioactivité naturelle "normale" est de $5 \mathrm{~Bq}$.

2.2.3.2. La quantité de ${ }^{230} \mathrm{Th}$ provenant des aliments est, sans doute, du même ordre de grandeur que celle de ${ }^{238} \mathrm{U}$, bien qu'il n'y ait pas d'information à ce sujet; de même, les teneurs des eaux de boisson ne sont pas connues.

2.2.3.3. Le radium 226 étant un alcalino-terreux, son comportement rappelle celui du calcium. En conséquence, une partie du radium provient des tissus végétaux et animaux, l'autre de la composante externe. En général, dans les régions sédimentaires, la quantité de radium présente dans l'eau est faible, mais on a trouvé en France [2] une activité de 2,7 Bq. I $^{-1}$ dans une eau de boisson. En France, la teneur en ${ }^{226} \mathrm{Ra}$ [3] des laits de grand mélange est toujours inférieure à $5 \mathrm{mBq} \mathrm{I}^{-1}$ lorsqu'ils proviennent des régions sédimentaires et supérieure à cette valeur lorsqu'ils proviennent de régions d'origine volcanique. L'ingestion annuelle dans une région "normale" est de $15 \mathrm{~Bq}$. En Inde, sur la côte du Kerala, l'absorption annuelle est de ${ }^{40} \mathrm{~Bq}$ et au Brésil, un petit groupe dont l'absorption annuelle se situe entre 140 et $540 \mathrm{~Bq}$ a été mis en évidence.

2.2.3.4. Le radon $\left({ }^{222} \mathrm{Rn}\right)$ et ses descendants à vie courte jusqu'à ${ }^{214} \mathrm{~Pb}$ ne représentent pas, en général, un problème dans l'alimentation. La teneur en $\mathrm{Rn}$ peut être très élevée dans l'eau de boisson, mais cet élément étant un gaz, il disparaît très rapidement dans l'organisme.

2.2.3.5. Les produits de filiation du radon à vie longue $-{ }^{210} \mathrm{~Pb},{ }^{210} \mathrm{Bi}$, ${ }^{210} \mathrm{Po}$ - existent dans le sol et dans l'air; cette fraction finit par se déposer sur la surface terrestre. Les végétaux sont donc contaminés par les deux voies. Dans les conditions normales, l'ingestion annuelle est d'environ $40 \mathrm{~Bq}$. Des concentrations élevées de 0,7 à $20 \mathrm{~Bq}$ de ${ }^{210} \mathrm{Po}$ par kilogramme existent dans la chair de poissons et de mollusques marins. Dans les régions arctiques, la viande de renne peut apporter $140 \mathrm{~Bq}$ de ${ }^{210} \mathrm{~Pb}$ et $1400 \mathrm{~Bq}$ de ${ }^{210} \mathrm{Po}$ par an à la population locale consommatrice de cet aliment. 


\subsubsection{Famille du thorium 232}

Considérée sous l'angle de la contamination alimentaire, cette famille peut être scindée en deux sous-familles : 1) ${ }^{232} \mathrm{Th}$ seul ; 2) ${ }^{228} \mathrm{Ra}$ à ${ }^{208} \mathrm{~Pb}$.

\subsubsection{Thorium ( $\left.{ }^{232} \mathrm{Th}\right)$}

Cet élément dans l'alimentation provient sans doute d'un dépôt d'origine externe, comme les radionucléides de la première sous-famille de l'uranium; on peut donc admettre que l'ingestion annuelle est à peu près la même, soit $5 \mathrm{~Bq}$.

2.2.4.2. Sous-famille du radium $228:{ }^{228} \mathrm{Ra},{ }^{228} \mathrm{Ac},{ }^{228} \mathrm{Th},{ }^{224} \mathrm{Ra},{ }^{229} \mathrm{Rn}$ à ${ }^{208} \mathrm{TI}$

Le radium étant assimilable plus facilement par les végétaux et les animaux que le thorium, l'activité retrouvée dans l'alimentation sera plus élevée que celle du thorium. Dans les régions à radioactivité normale, l'ingestion annuelle de ${ }^{228} \mathrm{Ra}$ est de $15 \mathrm{~Bq}$. Dans certaines régions de l'Inde et du Brésil, très actives, l'ingestion peut atteindre $3000 \mathrm{~Bq} \cdot \mathrm{an}^{-1}$.

\section{REFERENCES}

[1] UNSCEAR. Ionizing radiation: sources and biological effects, 1982 report to the general assembly, with annexes. New York: United Nations, 1982.

[2] REMY M.L., PELLERIN P. Quelques données générales sur la radioactivité des sources hydrominérales françaises. Colloque sur les eaux thermominérales. Bagnères-de-Luchon, 1981.

[3] DREUILLE M. de. Contribution à l'étude de la radioactivité naturelle du lait. Teneurs en radium 226. Thèse, Docteur Vétérinaire, Lyon, 1964. 